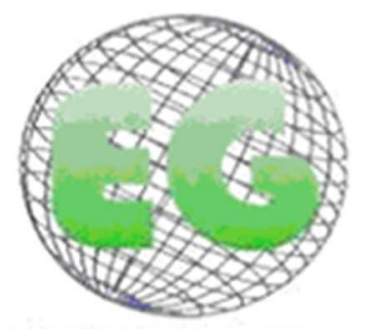

ISSN 1695-6141 $N^{\circ} 64$

\title{
ORIGINALES
}

\section{Asociación entre características sociodemográficas, síntomas depresivos, estrés y ansiedad en tiempos de la COVID-19}

Association between sociodemographic characteristics, depressive symptoms, stress and anxiety in times of COVID-19

\section{Pedro Enrique Trujillo-Hernández ${ }^{1}$ \\ Dafne Astrid Gómez-Melasio ${ }^{1}$ \\ Braulio Josué Lara-Reyes ${ }^{1}$ \\ Isaí Arturo Medina-Fernández ${ }^{1}$ \\ Eva Kerena Hernández-Martínez ${ }^{1}$}

1 Universidad Autónoma de Coahuila. Facultad de Enfermería "Dr. Santiago Valdés Galindo" Saltillo Coahuila, México. dafne.gomez@uadec.edu.mx

\section{https://doi.org/10.6018/eglobal.471511}

Recibido: $3 / 03 / 2021$

Aceptado: 24/04/2021

\section{RESUMEN:}

Introducción: La pandemia por la COVID-19 ha afectado la salud mental de la población. Los síntomas depresivos, estrés y ansiedad son respuestas negativas que pueden presentarse ante situaciones de riesgo en las que se hace frente a la incertidumbre, lo desconocido o situaciones de crisis, como la actual crisis sanitaria.

Objetivo: Identificar la asociación entre los factores sociodemográficos y síntomas depresivos, nivel de estrés y ansiedad en tiempos de la COVID-19 en habitantes de la ciudad de Saltillo Coahuila, México.

Métodos: Estudio correlacional predictivo de corte transversal. La población fueron habitantes de la ciudad de Saltillo Coahuila, México. La muestra fue de 501 participantes, recolectados a través de una encuesta online que se habilitó del 5 al 24 de octubre de 2020.

Resultados: La edad (OR = .93; 95\% IC: .900 - .979) y estar sin pareja (OR $=2.64 ; 95 \%$ IC: 1.347 5.201) aumenta la probabilidad de sufrir síntomas depresivos severos. Personas más jóvenes tuvieron .95 mayor probabilidad de presentar estrés elevado. Ser mujer (OR $=2.37 ; 95 \%$ IC: $1.144-4.915)$ y no contar con empleo (OR $=2.62$; $95 \%$ IC: 1.308 - 5.254) se asoció con mayor probabilidad de sufrir ansiedad severa.

Conclusión: Se encontró asociación en factores sociodemográficos como el sexo, edad e ingreso económico con los síntomas depresivos, nivel de estrés y ansiedad en tiempos de la COVID-19. Estos resultados proyectan datos preliminares de algunos aspectos relacionados con la salud mental en el contexto mexicano durante la actual crisis sanitaria.

Palabras clave: Infecciones por Coronavirus; Depresión; Estrés Psicológico; Ansiedad; Salud Mental.

\section{ABSTRACT:}

Introduction: The COVID-19 pandemic has affected the mental health of the population. Depressive symptoms, stress, and anxiety are negative responses that can occur in risky situations in which uncertainty, the unknown or crisis situations are faced, such as the current health crisis. 
Objective: To identify the association between sociodemographic factors and depressive symptoms, level of stress and anxiety in times of COVID-19 in inhabitants of the city of Saltillo Coahuila, Mexico.

Methods: Cross-sectional predictive correlational study. The population was inhabitants of the city of Saltillo Coahuila, Mexico. The sample consisted of 501 participants, who were collected through an online survey that was made available from October 5 to 24, 2020.

Results: Age (OR $=.93 ; 95 \% \mathrm{Cl}: .900-.979)$ and being without a partner (OR $=2.64 ; 95 \% \mathrm{Cl}: 1.347$ $5.201)$ increase the probability of suffering severe depressive symptoms. Younger people were .95 more likely to have elevated stress. Being a woman (OR $=2.37 ; 95 \% \mathrm{Cl}: 1,144-4,915)$ and not having a job $(\mathrm{OR}=2.62 ; 95 \% \mathrm{Cl}: 1,308-5,254)$ was associated with a higher probability of suffering from severe anxiety.

Conclusion: Associations were found in sociodemographic factors such as sex, age and economic income with depressive symptoms, level of stress and anxiety in times of COVID-19. These results project preliminary data on some aspects related to mental health in the Mexican context during the current health crisis.

Key words: Coronavirus Infections; Depression; Psychological Stress; Anxiety; Mental Health.

\section{INTRODUCCIÓN}

La enfermedad de un nuevo coronavirus denominado por la Organización Mundial de la Salud como la COVID-19 se ha convertido en una emergencia sanitaria(1). Este virus se identificó en diciembre de 2019 en Wuhan, provincia de Hubei, China. Las cepas de la COVID-19 se han relacionado con el coronavirus del Síndrome Respiratorio Agudo Severo (SARS-CoV) y el coronavirus del Síndrome Respiratorio del Medio Oriente (MERS-CoV) ${ }^{(2)}$, características por las cuales se ha considerado de alta peligrosidad para el ser humano.

A nivel mundial se han reportado más de 147.1 millones de contagios y ha superado 3.1 millones de defunciones a causa de la COVID-19(3). En México, se han identificado más de 2.3 millones de casos confirmados; 433,453 casos sospechosos y 214,947 defunciones. De los casos confirmados, $49.95 \%$ son mujeres y $50.05 \%$ son hombres, el $18.75 \%$ se encuentran hospitalizados y $81.25 \%$ son pacientes ambulatorios. Las principales comorbilidades asociadas son: hipertensión (17.21\%), obesidad $(14.26 \%)$, diabetes $(13.25 \%)$ y tabaquismo $(7.33 \%)^{(4)}$.

Debido a la alta incidencia de casos, su rápida propagación y gravedad, el 11 de marzo de 2020 la COVID-19 fue denominada como una pandemia(5). A partir de este comunicado los gobiernos del mundo, incluido México, implementaron diversas estrategias de contención de la COVID-19, tales como la cuarentena, aislamiento o distanciamiento social, entre otras. Medidas que afectan los patrones de conducta individual o colectivo, además de repercutir a nivel biopsicosocial y afectar la salud mental de la población ${ }^{(6-8)}$.

El distanciamiento entre las personas y la ausencia de comunicación interpersonal, la falta de vacunas y tratamientos efectivos, desconocer la duración del aislamiento y el alto impacto económico, social y sanitario generan incertidumbre en la población, y aumentan el riesgo de sufrir trastornos depresivos, estrés y ansiedad( ${ }^{(9-11)}$. Así mismo, se ha detectado un aumento de emociones negativas (ansiedad, depresión e indignación) y una disminución de emociones positivas (felicidad y satisfacción) ${ }^{(12)}$. En una pandemia se puede esperar que las personas experimenten miedo, estrés, ansiedad, angustia, irritabilidad y enojo, así como recuerdos de experiencias traumáticas, dificultad para concentrarse y problemas del sueño(13). 
La pandemia por la COVID-19 ha afectado la salud mental de muchas personas alrededor del mundo y ha demostrado, en cierta medida, la fragilidad que se puede llegar a tener ante eventos que ponen en riesgo la salud y vida de las personas. La pandemia por la COVID-19 ha sido reflejo de cómo una enfermedad física trasciende del área biológica para afectar el área mental, añadiendo manifestaciones psicológicas a la sintomatología común ${ }^{(14)}$.

Enfermería debe sobresalir ante los desafíos provocados por la pandemia de la COVID-19 e innovar en la profesión. Se han observado contribuciones del personal de enfermería en el contexto de la salud, acciones relevantes como: educación para la salud, prevención de infecciones y vigilancia epidemiológica, organización y preparación de servicios a largo plazo, protección de personas con enfermedades crónicas y en la actualidad en la atención de casos graves por la COVID-19(15-17).

Ante el contexto por la pandemia el rol de enfermería es crucial, debido a la necesidad de proveer cuidados directos, soporte emocional, educar a pacientes y personal de salud, abogar por los pacientes y sus familias. Además, es necesaria la coordinación de acciones de prevención en la comunidad, en donde se debe contemplar la salud mental, debido a que la alteración de esta puede llegar a incapacitar a las personas y afectar su calidad de vida(18).

Es decir, enfermería tiene un papel importante como promotor y gestor del cuidado de las personas para preservar sus vidas, esta labor no se limita a atender las afecciones biológicas sino a satisfacer a la vez las necesidades psicosociales. De igual manera, enfermería es responsable de brindar cuidados eficientes, efectivos y de calidad acorde a las dinámicas contemporáneas, a través del cuidado de las relaciones interpersonales, en donde se desempeñan diferentes roles como el de enseñanza y asesoramiento $^{(19,20)}$.

Es relevante que el profesional de enfermería contribuya a mejorar la salud mental en la población, en un inicio, con la producción de investigaciones que permitan explorar y conocer las principales enfermedades psicológicas presentes en la población en tiempos de la COVID-19 y los factores asociados a dichas afecciones. Posterior, desarrollar programas de entrenamiento y acompañamiento sobre cuidados enfocados en la implementación del apoyo educativo, mediante estrategias cognitivo conductuales con la finalidad de prevenir, controlar o disminuir las afecciones psicológicas durante esta emergencia sanitaria. Por lo tanto, el objetivo de este estudio fue identificar la asociación entre los factores sociodemográficos y síntomas depresivos, nivel de estrés y ansiedad en tiempos de la COVID-19 en habitantes de la ciudad de Saltillo Coahuila, México.

\section{MÉTODOS}

Se utilizó un diseño de estudio correlacional predictivo de corte transversal(21). La población fueron habitantes de la ciudad de Saltillo Coahuila, México. La muestra estuvo conformada por 501 participantes. Para la recolección de los datos se empleó un muestreo virtual online en redes sociales convencionales. La encuesta se habilitó del 5 al 24 de octubre de 2020. Tener al menos 18 años o más fue el único criterio de inclusión adoptado. 
Se realizó una cédula de datos personales (CDP) que incluía preguntas como edad, sexo, estado civil, ingreso económico, ocupación, enfermedades crónicas presentes, si perdió el trabajo o le redujeron el salario a causa de la pandemia y seguimiento de recomendaciones sanitarias de cuarentena como asilamiento o distanciamiento social. Para medir los síntomas depresivos se utilizó la Escala de Depresión del Centro de Estudios Epidemiológicos (CES-D20) de Radolff(22). Consta de 20 ítems, los parámetros de la escala son: $0=$ ningún día; $1=1$ a 3 días; $2=4$ a 6 días y $3=$ todos los días, el rango fluctúa entre 0 a 60 . Los puntos de corte son: 0 a 15 sin síntomas depresivos, 16 a 20 síntomas depresivos leves, 21 a 25 síntomas depresivos moderados y puntajes superiores o igual a 26 síntomas depresivos severos. Cada ítem refleja la frecuencia que fue experimentado en la última semana.

Las propiedades psicométricas del CES-D 20 son: a) una consistencia interna (alpha de Cronbach) de 0.85 para población general y 0.90 para pacientes psiquiátricos; b) confiabilidad test-retest de 0.54 para población general y 0.53 en pacientes psiquiátricos; c) el porcentaje de no respuesta es bajo, por lo que su aceptabilidad es alta. La escala es generalizable, pues el análisis por subgrupos contiene coeficientes alphas mayores de 0.80 .

Para evaluar el nivel de estrés se aplicó la Escala de Estrés Percibido (PSS-14) de Cohen, Kamarck y Mermelstein ${ }^{(23)}$, diseñada para medir el grado en que las situaciones de vida son percibidas como estresantes. Adaptada en México en su versión en español por González y Landero(24). El PSS-14 tiene una adecuada consistencia interna de 0.80 . Es un instrumento de auto informe que evalúa el nivel de estrés percibido durante el último mes, consta de 14 ítems, utiliza un formato de respuesta tipo likert de cinco puntos $(0=$ nunca, $1=$ casi nunca, $2=$ de vez en cuando, 3 = a menudo y 4 = muy a menudo).

El rango de la escala oscila de 0 a 56 , las puntuaciones altas indican mayor estrés percibido, en esta investigación se clasificó en nivel de estrés alto y bajo, se tomó de referencia la mediana del puntaje total de la escala. La escala PSS-14 ha demostrado ser fiable y válida para evaluar los síntomas de estrés en diversas poblaciones.

Para detectar los niveles de ansiedad se empleó la Escala del Trastorno de Ansiedad Generalizada (GAD-7) de Spitzer et al., ${ }^{(25)}$ diseñada en un inicio para la detección y medida de la severidad del trastorno de ansiedad generalizada, sin embargo, posee buenas características operativas para el trastorno de ansiedad social. La GAD-7 consta de 7 preguntas puntuables entre 0 y 3, con puntuaciones mínima y máxima posibles de 0 y 21. Los puntos de corte son: 0 - $4=$ No se aprecian síntomas de ansiedad; 5 - 9 = Se aprecian síntomas de ansiedad leves; 10 - $14=$ Se aprecian síntomas de ansiedad moderados y 15 - 21 = Se aprecian síntomas de ansiedad severos. La GAD-7 ha reportado coeficiente alpha de Cronbach de 0.93. Con el corte de 10 puntos, los valores de sensibilidad fueron de $86.80 \%$ y especificidad de $93.40 \%$. La encuesta digital fue creada por los investigadores en la plataforma QuestionPro. Las preguntas de las escalas fueron adaptadas para hacer referencia al tiempo que ha estado en cuarentena o aislamiento social a causa de la COVID-19. Posterior, el enlace de la encuesta fue compartido a través de redes sociales virtuales convencionales (Facebook, Messenger, Linkedln y WhatsApp) mediante diversos grupos en algunas de ellas. Al acceder a la encuesta, se presentó en primera instancia el consentimiento informado con una descripción detallada del estudio, así como los correos de los investigadores para responder cualquier duda que se pudiera 
presentar. Las personas que aceptaron participar dieron su consentimiento informado electrónico y continuaron con las preguntas de la encuesta. Al enviar sus respuestas se les agradeció por su tiempo y de esa forma concluyó su participación. El orden de aparición de los instrumentos en la plataforma fue el siguiente: 1) CDP, 2) CES-D20, 3) PSS-14 y 4) GAD-7. El tiempo aproximado de llenado fue de 10 - 15 minutos y para garantizar el anonimato no se recopilaron datos personales que pudieran permitir la identificación de los participantes.

Para caracterizar la muestra de estudio se utilizaron análisis descriptivos (medidas de tendencia central, frecuencias y porcentajes). Se obtuvieron alfas de Cronbach para obtener la fiabilidad de los instrumentos. Con la finalidad de determinar la asociación entre las variables sociodemográficas y los niveles de síntomas depresivos, estrés y ansiedad se realizó análisis de regresión logística binaria y multinomial, a través de la prueba de Chi de Pearson y $\mathrm{R}^{2}$ de Nagelkerke, que explican la significancia de las variables y el porcentaje de la varianza explicada.

El presente estudio se apegó a los lineamientos del Reglamento de la Ley General en Materia de Investigación para la Salud(26), Título Segundo de los aspectos éticos de la investigación en seres humanos, a fín de asegurar el respeto de la dignidad de los sujetos, la protección de sus derechos y el uso de consentimiento informado. Además, se siguieron las recomendaciones éticas contenidas en la Declaración de Helsinki ${ }^{(27)}$.

\section{RESULTADOS}

La edad media fue $32.69(D E=10.32), 68.50 \%$ pertenecen al sexo femenino y $31.50 \%$ masculino. El $46.90 \%$ eran solteros, $39.10 \%$ casados y el $8.40 \%$ mantenía una relación en unión libre. Se encontró que el $25.10 \%$ sufre sobrepeso/obesidad (SP-OB), 6.00\% hipertensión (HTA) y 5.00\% diabetes (DM). En el ingreso económico mensual individual, $28.90 \%$ percibe ingresos inferiores a 5 mil pesos mexicanos (MN), $33.70 \%$ recibe de 5,001 a 10 mil $\mathrm{MN}$ y solo $12.40 \%$ mencionó percibir ingresos superiores a 20 mil MN.

En el ingreso económico mensual familiar, 26.70\% reportó ingresos de 5,001 a 10 mil MN, seguido del $25.70 \%$ que mencionó ingresos superiores a 20 mil MN. En cuanto a la cobertura médica de los participantes, $66.50 \%$ señaló ser derechohabiente del Instituto Mexicano del Seguro Social (IMSS), 13.20\% del Instituto de Seguridad y Servicios Sociales de los Trabajadores del Estado (ISSSTE), 8.60\% cuenta con seguro médico privado y el $7.60 \%$ indicó no tener ningún servicio de atención de salud.

Cabe destacar que los salarios reportados por los participantes oscilan entre 1 a 2 salarios mínimos en el contexto mexicano, el cual es de 141.70 pesos MN diarios, lo que equivale aproximadamente a 7 dólares americanos o 5.82 euros por día. Estos salarios son insuficientes pues la contingencia sanitaria puede ocasionar gastos relacionados a la salud o a la prevención de la COVID-19, los cuales pueden ascender a más de 16 mil pesos MN en pacientes ambulatorios, mientras que para los pacientes que requieren el ingreso a unidades de cuidados intensivos en hospitales privados se ha reportado cantidades superiores a un millón de pesos MN. 
Así mismo, el sistema nacional de salud está conformado por instituciones públicas y privadas. En instituciones públicas como el IMSS e ISSSTE se aportan contribuciones por el trabajador, patrón y el gobierno federal. En cuanto a la cobertura sanitaria nacional se observa que: 51 millones de personas pertenecen al IMSS, 35.5 millones al Instituto de Salud para el Bienestar, 8.8 millones al ISSSTE y 2.8 millones tienen seguridad privada ${ }^{(28)}$.

Con relación a la ocupación, $65.40 \%$ son trabajadores de alguna empresa o institución, $15.20 \%$ son estudiantes y $10.40 \%$ realizan actividades del hogar. El $22.60 \%$ manifestó que él o algún familiar perdieron su empleo a causa de la pandemia COVID-19. 53.70\% refirió que se quedó en casa y no fue a laborar por indicaciones de su lugar de trabajo, el $36.50 \%$ ha realizado actividades laborales desde su casa. Por último, $35.70 \%$ dijo que su salario había disminuido a causa la situación generada por las medidas de contención de la COVID-19.

En cuanto a las patologías que sufren las personas que viven con los participantes y que son considerados factores de riesgo ante la COVID-19, 26.90\% padece DM, $30.10 \%$ HTA, $34.70 \%$ SP-OB, $7 \%$ problemas respiratorios y $25.70 \%$ viven con personas mayores de 60 años.

Respecto a los síntomas depresivos ( $\mathrm{M}=13.05$; $D E=9.75), 64.70 \%$ manifestó no tenerlos, $12.80 \%$ presentó síntomas depresivos leves, y el $11.40 \%$ y $11.20 \%$ de moderados a severos. Referente al nivel de estrés $(M=21.81 ; D E=8.94), 51.90 \%$ reportó altos niveles. Con relación al nivel de ansiedad $(\mathrm{M}=7.12 ; D E=5.08), 42.90 \%$ tuvo ansiedad leve, $32.10 \%$ no presentó ansiedad, $13.60 \%$ reportó ansiedad moderada y $11.40 \%$ ansiedad severa. Los análisis de fiabilidad (alpha de Cronbach [a]) de las escalas utilizadas demostraron valores aceptables en la muestra de estudio (CES-D20 $\alpha=0.92 ;$ PSS-14 $\alpha=0.86$ y GAD-7 $\alpha=0.92$ ). En la tabla 1 se presenta la relación entre los síntomas depresivos, nivel de estrés y ansiedad y factores sociodemográficos.

Tabla 1. Análisis de Chi cuadrado: Síntomas depresivos, estrés y ansiedad y factores sociodemográficos

\begin{tabular}{|c|c|c|c|}
\hline & Síntomas depresivos & Nivel de Estrés & Nivel de Ansiedad \\
\hline Edad & .68 & $.01^{*}$ & .18 \\
\hline Sexo & .29 & .44 & .07 \\
\hline Estado Civil & $.01^{*}$ & $.01^{* *}$ & .20 \\
\hline Ocupación & $.01^{* *}$ & $.02^{*}$ & .09 \\
\hline Pérdida del empleo & $.01^{* *}$ & $.01^{* *}$ & $.01^{* *}$ \\
\hline Distanciamiento social & .43 & .54 & .45 \\
\hline Disminución de salario & $.02^{*}$ & $.03^{*}$ & 23 \\
\hline
\end{tabular}

Nota: $p=\leq .05^{*}, p=\leq .01^{* *}$

Para identificar los factores sociodemográficos asociados a los síntomas depresivos derivados de la pandemia COVID-19, se realizó un análisis de regresión logística multinomial. El modelo fue significativo $\left(X^{2}=58.877, g l=15, p=<.001\right)$. La prueba de chi cuadrada de Pearson $\left(X^{2}=718.606, g l=702, p=<.324\right)$ y la desvianza de chi cuadrada $\left(X^{2}=551.947, g l=702, p=1.000\right)$ indicaron un buen ajuste del modelo. La $\mathrm{R}^{2}$ de Nagelkerke fue de .127 , que predice el $12.70 \%$ de la varianza. El modelo mostró que los participantes más jóvenes presentaron .93 (95\% IC: .900 - .979) más probabilidad de sufrir síntomas depresivos moderados. En las personas sin pareja 
$(\mathrm{OR}=2.64 ; 95 \%$ IC: $1.347-5.201)$, desempleadas $(\mathrm{OR}=2.43 ; 95 \%$ IC: $1.330-$ 4.453) y que perdieron él o algún familiar su trabajo a causa de la pandemia COVID19 (OR $=.35 ; 95 \%$ IC: $.191-.664)$ aumentó la probabilidad de sufrir síntomas depresivos severos.

Para observar la asociación entre las variables sociodemográficas y el nivel de estrés, se desarrolló un análisis de regresión logística binaria. El análisis indica que el modelo nulo tiene un $52 \%$ de probabilidad, el modelo fue significativo $\left(X^{2}=60.570, g l=6, p=\right.$ $<.001)$. La prueba Hosmer y Lemeshow $\left(X^{2}=3.477, g l=8, p=<.901\right)$ indica un buen ajuste del modelo. La $\mathrm{R}^{2}$ de Nagelkerke fue de .148 , que predice el $14.80 \%$ de la varianza. Con la clasificación se observa que el modelo predice el $62.60 \%$ en comparación con el modelo nulo. Se encontró que los participantes más jóvenes tuvieron .95 mayor probabilidad de presentar niveles de estrés elevados. Y aquellos que no perdieron su empleo a causa de la pandemia presentaron .37 menor probabilidad de tener niveles estrés elevado.

Se realizó un análisis de regresión logística multinomial para identificar las variables sociodemográficas asociadas al nivel de ansiedad. El modelo fue significativo $\left(X^{2}=\right.$ 64.442, $g l=27, p=<.001)$. La prueba de Chi de Pearson $\left(X^{2}=1111.84, g l=1095, p=\right.$ $<.355)$ y la desvianza de Chi cuadrada $\left(X^{2}=921.180, g l=1095, p=1.000\right)$ indicaron un buen ajuste del modelo. La $\mathrm{R}^{2}$ de Nagelkerke fue de .132, que predice el $13.20 \%$ de la varianza. Se detectó que si el participante o algún familiar perdieron su empleo a causa de la pandemia tuvieron .49 (95\% IC: .283 - .865) mayor probabilidad de presentar niveles de ansiedad leve. La edad se asoció de forma negativa con el nivel de ansiedad, de modo que las personas con menor número de años tuvieron .94 (95\% IC: .916 - .983) mayor probabilidad de presentar niveles de ansiedad moderada. Ser mujer (OR = 2.48; 95\% IC: $1.254-4.941)$ y no tener pareja (OR = 1.95; 95\% IC: 1.015 - 3.774) se asoció con mayor probabilidad de presentar niveles de ansiedad moderada.

Asimismo, pertenecer al sexo femenino (OR $=2.37 ; 95 \%$ IC: $1.144-4.915)$ y no contar con empleo (OR $=2.62 ; 95 \%$ IC: 1.308 - 5.254) se relacionó con mayor probabilidad de sufrir niveles de ansiedad severa. La pérdida del trabajo del participante o de algún familiar a causa de la pandemia (OR $=.31 ; 95 \%$ IC: $.149-$ .654) y referir ingresos económicos familiares menores de 5 mil MN (OR $=.23 ; 95 \%$ IC: .068 - .812) o entre 5001 y 10 mil MN (OR = .29; 95\% IC: .119 - .747) se asoció con mayor probabilidad de presentar niveles de ansiedad severa.

\section{DISCUSIÓN}

El presente trabajo de investigación identificó asociaciones entre factores sociodemográficos y síntomas depresivos, nivel de estrés y ansiedad en tiempos de pandemia por la COVID-19. Los resultados demuestran datos similares a los reportados por Lei et al., ${ }^{(29)}$ que encontraron niveles altos de síntomas depresivos $(50.70 \%)$, ansiedad (44.70\%) y estrés $(73.40 \%)$. Datos similares a los Elbay et al., ${ }^{(30)}$ en donde, $64.70 \%$ tenía síntomas depresivos, $51.60 \%$ tenía síntomas de ansiedad y $41.20 \%$ tenía síntomas relacionados con el estrés. Y a los de Wang et al., ${ }^{(31)}$ que observaron, en más de la mitad de los encuestados estrés psicológico de moderado a severo y cerca de un tercio reportó ansiedad de moderada a severa. 
También, se encontraron relaciones significativas mediante chi cuadrada entre las variables de interés con algunos factores sociodemográficos (edad, estado civil, ocupación, perdida del empleo y disminución de salario). Estos resultados difieren de algunas investigaciones que demuestran que aparte de los factores antes mencionados, el padecer enfermedades crónicas degenerativas se relacionaban con los síntomas depresivos, estrés y ansiedad ${ }^{(32,33)}$, característica que en este estudio no se encontró, lo cual se puede atribuir al bajo porcentaje de enfermedades crónicas en la muestra de estudio. Sin embargo, Parrado-González y León-Jariego ${ }^{(34)}$ mencionan que ser mujer y tener menor nivel de ingresos económicos, presentaron mayor impacto psicológico y peor salud mental.

Una posible causa es el poder adquisitivo que se tiene en el país, siendo México un país en vías de desarrollo, la cuestión económica y sociocultural impacta de manera considerable, al igual que otros países de América Latina. Esto se puede corroborar con los resultados de Johnson et al., ${ }^{(35)}$ donde señalan que el impacto en la salud mental por la COVID-19 es desigual según el género, el nivel educativo y el confort percibido en el hogar, además de las consecuencias sociales y económicas que supone la medida del aislamiento para la vida cotidiana, social y laboral.

Además, la Comisión Económica para América Latina y el Caribe ${ }^{(36)}$ señala que a causa de la COVID-19 la situación social se deteriora, debido al aumento de los índices de pobreza y pobreza extrema, la persistencia de las desigualdades y el descontento generalizado. Es decir, la crisis sanitaria tendrá repercusiones negativas en la salud y la educación, así como en el empleo y la pobreza, factores que se han relacionado como riesgo a sufrir síntomas depresivos, estrés y ansiedad, las cuales afectan la salud mental.

Respecto a las asociaciones identificadas entre las variables sociodemográficas y los síntomas depresivos en esta investigación, los participantes más jóvenes y en las personas sin pareja son los que presentaron mayor riesgo. Datos similares a lo reportado por Lei et al., ${ }^{(29)}$ en donde indican que el grupo de edad más joven tenía síntomas depresivos más altos y significativos que el grupo de más edad, además, el no tener alguna relación sentimental se relacionó con sufrir síntomas depresivos.

Lozano-Vargas ${ }^{(37)}$ encontró que las personas de más edad presentaron niveles altos de estrés psicológico, lo cual difiere en esta investigación, ya que se encontró que los participantes más jóvenes tuvieron .95 mayor probabilidad de presentar niveles de estrés elevados. Se ha reportado que las mujeres tienen dos veces más posibilidad de sufrir de estrés y ansiedad(38). El género femenino ha sido identificado como predictor de los síntomas del trastorno de estrés postraumático después de las pandemias ${ }^{(25)}$. Lo anterior concuerda con los resultados de esta investigación, en donde, ser mujer se asoció con mayor probabilidad de presentar niveles de ansiedad moderada.

Una posible explicación es que los jóvenes al tener un cambio repentino en sus actividades académicas, sociales y de diversa índole a causa del aislamiento, provocó cambios negativos en los patrones de conducta afectando la salud mental. En las mujeres, la probabilidad de presentar mayores niveles de ansiedad puede ser atribuible a la carga emocional del rol protector que desarrolla al cuidar de la familia o la sobrecarga de actividades domésticas que trae consigo el que los miembros de la 
familia estén más tiempo en casa. Más aun, se ha reportado que el factor hormonal puede influir y más si están expuestos a un peligro o riesgo como lo es la COVID-19.

Choi et al., ${ }^{(39)}$ encontraron que los participantes con ingreso económico familiar medio-alto presentaron niveles de ansiedad más bajos que las familias con ingreso económico medio-bajo. Datos similares a los de esta investigación, pues perder su trabajo y referir ingreso económico familiar menor de 10 mil MN se asoció con mayor probabilidad de presentar niveles de ansiedad severa. La pérdida del empleo trae consigo la pérdida del ingreso económico, situación que puede generar elevados niveles de ansiedad por no poder cubrir sus necesidades, y puede complicarse en caso de tener dependientes económicos.

Los resultados presentados permitieron conocer posibles enfermedades presentes en la población a causa de la cuarentena por la COVID-19 como síntomas depresivos, estrés y ansiedad, y algunas características sociodemográficas que se asociaron a dichas afecciones. Se espera que los hallazgos de este estudio puedan ser útiles para el personal de enfermería y sirvan como sustento en futuras investigaciones que permitan ampliar el conocimiento existente y así, desarrollar programas para promover la salud mental. El personal de enfermería debe hacer frente a los desafíos generados por la pandemia actual, innovar en el ejercicio profesional y posicionarse en la comunidad científica y población en general, para fortalecer las prácticas del cuidado en la salud mental mediante la e-Health, el uso de TIC's y el trabajo interdisciplinario $^{(14)}$.

Una de las principales limitaciones de esta investigación es que debido a la naturaleza transversal de la investigación, no fue posible observar los cambios en las variables de interés a lo largo del tiempo. Además, por el momento no es posible aplicar otro método de muestreo que no sea virtual online, debido a las medidas de contención de la pandemia. Por lo tanto, existe la posibilidad de sesgo de selección, ya que las personas sin acceso a Internet, a un dispositivo o a las redes sociales virtuales, no pudieron ser incluidas en este estudio. Sin embargo, los hallazgos pueden ser útiles para la comunidad científica y sanitaria para poder establecer los planes de acción en donde los factores contextuales y personales deben ser considerados y relacionados con la salud mental.

\section{CONCLUSIÓN}

Se encontró asociación con la edad, ingreso económico y el sexo con las diferentes variables psicológicas de interés (síntomas depresivos, nivel de estrés y ansiedad) en tiempos de la COVID-19. Se exhorta a los profesionales de salud a considerar la salud mental como una prioridad para sobrellevar esta crisis sanitaria. Según las proyecciones, la pandemia puede extenderse varios años y afectar la salud mental de las personas que se encuentran aún en confinamiento y aislamiento social. Estos resultados proyectan datos preliminares en el contexto mexicano, es necesario el desarrollo de intervenciones para reducir los síntomas depresivos, estrés y ansiedad. Por último, se deben realizar más estudios a gran escala en diferentes contextos de México incluidas áreas rurales e incorporar otros factores que puedan explicar mejor el fenómeno de estudio. 


\section{REFERENCIAS}

1. Organización Mundial de la Salud. Preguntas y respuestas sobre la enfermedad por coronavirus (COVID-19); World Helth Organization. [Internet]. 2020. [acceso: 15/11/2020]. Disponible en: https://www.who.int/es/emergencies/diseases/novelcoronavirus-2019/advice-for-public/q-a-coronaviruses

2. Zhou, P., Yang, X. L., Wang, X. G., Hu, B., Zhang, L., Zhang, W., et al. A pneumonia outbreak associated with a new coronavirus of probable bat origin. Nature. [Internet]. 2020. [acceso: 15/11/2020]; 579 (7798): $270 . \quad$ Disponible en:https://doi.org/10.1038/s41586-020-2012-7

3. Johns Hopkins University. COVID-19 Dashboard by the Center for Systems Science and Engineering (CSSE) at Johns Hopkins University (JHU)". ArcGIS. [Internet]. 2021. [acceso: 26/04/2021]. Disponible en: https://coronavirus.jhu.edu/map.html

4. Secretaria de Salud. Covid-19 México: Información General. [Internet]. 2021. [acceso: 26/04/2021]. Disponible en: https://datos.covid-19.conacyt.mx

5. Organización Mundial de la Salud. WHO Director-General's Opening Remarks at the Media Briefing on COVID-19-11. World Helth Organization. [Internet]. 2020. [acceso: 15/11/2020] Disponible en: https://www.who.int/dg/speeches/detail/whodirector-general-s-opening-remarks-at-the-media-briefing-on-covid-19---11-may-2020

6. American Psychological Association. Pandemics. [Internet]. 2020. [acceso: 25/10/2020]. Disponible en: https://www.apa.org/practice/programs/dmhi/researchinformation/pandemics

7. Brooks, S. K., Webster, R. K., Smith, L. E., Woodland, L., Wessely, S., Greenberg, N., et al. The psychological impact of quarantine and how to reduce it: rapid review of the evidence. Lancet. [Internet]. 2020. [acceso: 26/10/2020]. 14; 395 (10227): 912. Disponible en: https://doi.org/10.1016/S0140-6736(20)30460-8

8. Lippi, G., Henry, B., Bovo, C., Sanchis-Gomar, F. Health risks and potential remedies during prolonged lockdowns for coronavirus disease 2019 (COVID-19). Diagnosis. [Internet]. 2020. [acceso: 26/10/2020]. 26;7(2):85-90. Disponible en: https://www.degruyter.com/document/doi/10.1515/dx-2020-0041/html

9. Johnson, M. C., Saletti-Cuesta, L., Tumas, N. Emociones, preocupaciones y reflexiones frente a la pandemia del COVID-19 en Argentina. Cien Saude Colet. [Internet]. 2020. [acceso: 27/10/2020]. 25 (suppl 1): 2447. Disponible en: https://doi.org/10.1590/1413-81232020256.1.10472020

10. Xiao C. A Novel Approach of Consultation on 2019 Novel Coronavirus (COVID19)-Related Psychological and Mental Problems: Structured Letter Therapy. Psychiatry Investig. [Internet]. 2020. [acceso: 27/10/2020]. 17(2):175. Disponible en: https://doi.org/10.30773/pi.2020.0047

11. Zandifar A, Badrfam R. Iranian mental health during the COVID-19 epidemic. Asian J Psychiatr. [Internet]. 2020. [acceso: 28/10/2020]. 51:101990. Disponible en: https://doi.org/10.1016/i.ajp.2020.101990

12. Ho CS, Chee CY, Ho RC. Mental Health Strategies to Combat the Psychological Impact of COVID-19 Beyond Paranoia and Panic. Ann Acad Med Singap. [Internet]. 2020. [acceso: 27/10/2020]. 16;49(3):155. Disponible en: https://pubmed.ncbi.nlm.nih.gov/32200399/

13. Taylor, S. The psychology of pandemics: Preparing for the next global outbreak of infectious disease. Cambridge Scholars Publishing. [Internet]. 2019. Disponible en: https://cambridgescholars.com/product/978-1-5275-3959-4 
14. Martinez-Esquivel D. Desafíos para la enfermería de salud mental después del COVID-19. Rev. cienc. cuidad. [Internet]. 2020 [acceso 08/02/2021]. 17(3):122-9. Disponible en: https://doi.org/10.22463/17949831.2413

15. Chen SC, Lai YH, Tsay SL. Nursing perspectives on the impacts of COVID-19. J Nurs Res. [Internet]. 2020. [acceso: 27/04/2021]. 28(3). Disponible en: https://doi.org/10.1097/NRJ.0000000000000389

16. Courtenay M, Burnett E, Castro-Sanchez E, Figueiredo RM, Toit B, Gallagher R, et al. Preparing nurses for COVID-19 response efforts through involvement in antimicrobial stewardship programmes. J Hosp Infect. [Internet]. 2020. [acceso: 27/04/2021]. 106(1):176-8. Disponible en: https://doi.org/10.1016/j.jhin.2020.06.011

17. Osingada CP., Porta CM. Nursing and Sustainable Development Goals (SDGs) in a COVID-19 world: the state of the science and a call for nursing to lead. Public Health Nurs. [Internet]. 2020. [acceso: 28/04/2021]. 37(5):799-805. Disponible en: https://doi.org/10.1111/phn.12776

18. Stirling B., Hatcher J., Harmston J. Communicating the Changing Role of a Nurse in an Epidemic: The Example of the MERS-CoV Outbreak in Saudi Arabia. J Healthc Commun. [Internet]. 2017. [acceso: 28/04/2021]. 02(03). Disponible en: http://doi.org/10.4172/2472-1654.100070

19. Lluch Canut MT. Enfermería de salud mental: El arte y la ciencia del cuidar. Revista española de enfermería de salud mental. [Internet]. 2018. [acceso 09/02/2021]. 4: 2-3. Disponible en: http://doi.org/10.35761/reesme.2018.4.01

20. Peplau H. Relaciones interpersonales en Enfermería. Un marco de referencia conceptual para la Enfermería psicodinámica. Barcelona: Salvat Editores S.A.; 1990.

21. Gray, J. R., Grove, S. K., Sutherland, S. Burns and Grove's. The Practice of Nursing Research: Appraisal, Synthesis, and Generation of Evidence. Elsevier Health Sciences. [Internet]. 2017. Disponible en: https://www.elsevier.com/books/burns-andgroves-the-practice-of-nursing-research/gray/978-0-323-37758-4

22. Radloff, L. S. The CES-D scale: A self-report depression scale for research in the general population. Applied psychological measurement. [Internet]. 1977. [acceso: 29/10/2020]. 1(3), 385. Disponible en: https://doi.org/10.1177/014662167700100306

23. Cohen, S., Kamarck, T., Mermelstein, R. A global measure of perceived stress. Journal of health and social behavior, [Internet]. 1983. [acceso: 29/10/2020]. 24(4), 385. Disponible en: https://doi.org/10.2307/2136404

24. González, M. T., Landero, R. Factor structure of the Perceived Stress Scale (PSS) in a sample from Mexico. Spanish Journal of Psychology. [Internet]. 2007. [acceso: 29/10/2020]. 10(1), 199. Disponible en: https://pubmed.ncbi.nlm.nih.gov/17549893/

25. Spitzer RL, Kroenke K, Williams JB, Löwe B. A brief measure for assessing generalized anxiety disorder: the GAD-7. Arch Intern Med. [Internet]. 2006. [acceso: 30/10/2020]. 22;166(10):1092.

https://doi.org/10.1001/archinte.166.10.1092

26. Secretaria de Salud. Reglamento de la ley general de salud en materia de investigación para la salud. 1987 [Internet]. [acceso: 03/11/2020]. Disponible en: http://mail.repssguerrero.gob.mx/ipo2019/transparencia/3er trim/Frac I/REGLAMENT O DE LA LEY GENERAL DE SALUD EN MATERIA DE PROTECCION SOCIA L EN SALUD.pdf

27. World Medical Association. Declaración de Helsinki - Principios éticos para las investigaciones médicas en seres humanos. $64^{\text {a }}$ Asamblea General. Fortaleza, Brasil. 2013. [Internet]. [acceso: 05/11/2020]. Disponible en: http://repositorio.mederi.com.co/bitstream/handle/123456789/386/DeclaracionHelsinki-2013-Esp.pdf?sequence=1 
28. Instituto Nacional de Estadística y Geografía (INEGI). Salud y seguridad social: Derechohabiencia. [Internet]. 2020. [acceso: 28/04/2021]. Disponible en: https://inegi.org.mx/temas/derechohabiencia/

29. Lei, L., Huang, X., Zhang, S., Yang, J., Yang, L., Xu, M. Comparison of prevalence and associated factors of anxiety and depression among people affected by versus people unaffected by quarantine during the COVID-19 epidemic in southwestern China. Med Sci Monit. [Internet]. 2020. [acceso: 15/11/2020]. 26: e924609-1. Disponible en: https://doi.org/10.12659/MSM.924609.

30. Elbay RY, Kurtulmuş A, Arpacıoğlu S, Karadere E. Depression, anxiety, stress levels of physicians and associated factors in Covid-19 pandemics. Psychiatry Res. [Internet]. 2020. [acceso: 15/11/2020]. 290:113130. Disponible en: https://doi.org/10.1016/i.psychres.2020.113130

31. Wang C, Pan R, Wan X, Tan Y, Xu L, Ho CS, et al. Immediate Psychological Responses and Associated Factors during the Initial Stage of the 2019 Coronavirus Disease (COVID-19) Epidemic among the General Population in China. Int J Environ Res Public Health. [Internet]. 2020. [acceso: 25/11/2020] 6;17(5):1729. Disponible en: https://doi.org/10.3390/ijerph17051729

32. Dong XC, Li JM, Bai JY, Liu ZQ, Zhou PH, Gao L, et al. Epidemiological characteristics of confirmed COVID-19 cases in Tianjin. Zhonghua Liu Xing Bing Xue Za Zhi. [Internet]. 2020. [acceso: 06/12/2020]. 10;41(5):638-641. Disponible en: https://doi.org/10.3760/cma.j.cn112338-20200221-00146.

33. Ozamiz-Etxebarria N, Dosil-Santamaria M, Picaza-Gorrochategui M, IdoiagaMondragon N. Stress, anxiety, and depression levels in the initial stage of the COVID19 outbreak in a population sample in the northern Spain. Cad Saude Publica. [Internet]. 2020. [acceso: 22/12/2020]. 30;36(4):e00054020. Disponible en: https://doi.org/10.1590/0102-311X00054020

34. Parrado-González A, León-Jariego JC. COVID-19: Factores asociados al malestar emocional y morbilidad psíquica en población española. Rev Esp Salud Pública. [Internet]. 2020. [acceso: 07/02/2021]. 94: 8 e202006058. Disponible en: https://pubmed.ncbi.nlm.nih.gov/32507849/

35. Johnson MC, Saletti-Cuesta L, Tumas N. Emociones, preocupaciones $y$ reflexiones frente a la pandemia del COVID-19 en Argentina. Ciência \& Saúde Coletiva. [Internet]. 2020. [acceso: 09/02/2021]. 25, 2447-2456. Disponible en: https://doi.org/10.1590/1413-81232020256.1.10472020

36. Comisión Económica para América Latina y el Caribe. América Latina y el Caribe ante la pandemia del COVID-19: efectos económicos y sociales. 2020. [Internet]. [acceso: 08/02/2021]. Disponible en: https://repositorio.cepal.org/handle/11362/45337 37. Lozano-Vargas, A. Impacto de la epidemia del Coronavirus (COVID-19) en la salud mental del personal de salud y en la población general de China. Rev de Neuropsiquiatr. [Internet]. 2020. [acceso: 20/12/2020]. 83(1), 51. Disponible en: http://dx.doi.org/10.20453/rnp.v83i1.3687

38. Foa EB, Street GP. Women and traumatic events. J Clin Psychiatry. [Internet]. 2001. [acceso: 28/12/2020]. 62 Suppl 17:29-34. Disponible en: https://pubmed.ncbi.nlm.nih.gov/11495093/

39. Choi EPH, Hui BPH, Wan EYF. Depression and Anxiety in Hong Kong during COVID-19. Int J Environ Res Public Health. [Internet]. 2020. [acceso: 28/12/2020] 25;17(10):3740. Disponible en: https://doi.org/10.3390/ijerph17103740 
